\title{
DEEP ROSAT SURVEYS \& THE CONTRIBUTION OF AGNS TO THE SOFT X-RAY BACKGROUND
}

\author{
I. Lehmann ${ }^{1}$, G. Hasinger ${ }^{1}$, M. Schmidt ${ }^{2}$, J.E. Gunn ${ }^{3}$, D.P. Schneider ${ }^{4}$, \\ R. Giacconi ${ }^{5}$, M. McCaughrean ${ }^{1}$, J. Trümper ${ }^{6}$ and G. Zamorani ${ }^{7,}{ }^{8}$ \\ 1) Astrophysikalisches Institut Potsdam (AIP), An der Sternwarte 16, D-14482 Pots- \\ dam, Germany \\ 2) California Institute of Technology, Pasadena, CA 91125, USA \\ 3) Princeton University Observatory, Peyton Hall, Princeton, NJ 08540, USA \\ 4) Department of Astronomy and Astrophysics, The Pennsylvania State University, Uni- \\ versity Park, PA 16802, USA \\ 5) Associated Universities, Inc.1400 16th Street, NW, Suite 730 Washington, DC 20036, \\ USA \\ 6) Max-Planck-Institut für extraterrestrische Physik, Karl-Schwarzschild-Str. 2, D-85748 \\ Garching bei München, Germany \\ 7) Osservatorio Astronomico, Via Ranzani 1, 1-40127, Bologna, Italy \\ 8) Istituto di Radioastronomia del CNR, via Gobetti 101, I-40129, Bologna, Italy
}

\begin{abstract}
The ROSAT Deep Surveys in the Lockman Hole have revealed that AGNs are the main contributors $(\sim 75 \%)$ to the soft $\mathrm{X}$-ray background in the 1-2 keV band. Using new optical/infrared and radio observations we have obtained a nearly complete identification (93\%) of the $91 \mathrm{X}$-ray sources down to a limiting flux of $1.2 \cdot 10^{-15} \mathrm{erg} \mathrm{cm}^{-2} \mathrm{~s}^{-1}$ in the $0.5-2.0 \mathrm{keV}$ band. We present the optical colours and the emission line properties of our AGNs in comparison with other X-ray selected AGN samples. Furthermore we discuss the fraction of red AGNs found in the ROSAT Deep Surveys. From the ROSAT Deep Surveys we see no evidence for a new class of X-ray bright galaxies, which significantly contributes to the soft X-ray background.
\end{abstract}

KEYWORDS: Surveys-galaxies: active-Galaxies: Seyferts-quasars: emission lines-X-rays: galaxies

\section{INTRODUCTION}

Deep X-ray surveys and the optical/infrared identification of the detected faint Xray sources are the key to understand the origin of the $\mathrm{X}$-ray background, discovered more than thirty years ago (Giacconi et al. 1962). We have performed the most sensitive soft X-ray survey (to date) in the Lockman Hole (Hasinger et al. 1993), a region of extremely low galactic hydrogen column density (Lockman et al. 1986).

The Deep ROSAT Surveys in the Lockman Hole consist of a $207 \mathrm{ksec}$ ROSAT

CP599, X-Ray Astronomy, edited by N. E. White et al. (C) 2001 American Institute of Physics 0-7354-0043-1/01/\$18.00

189 


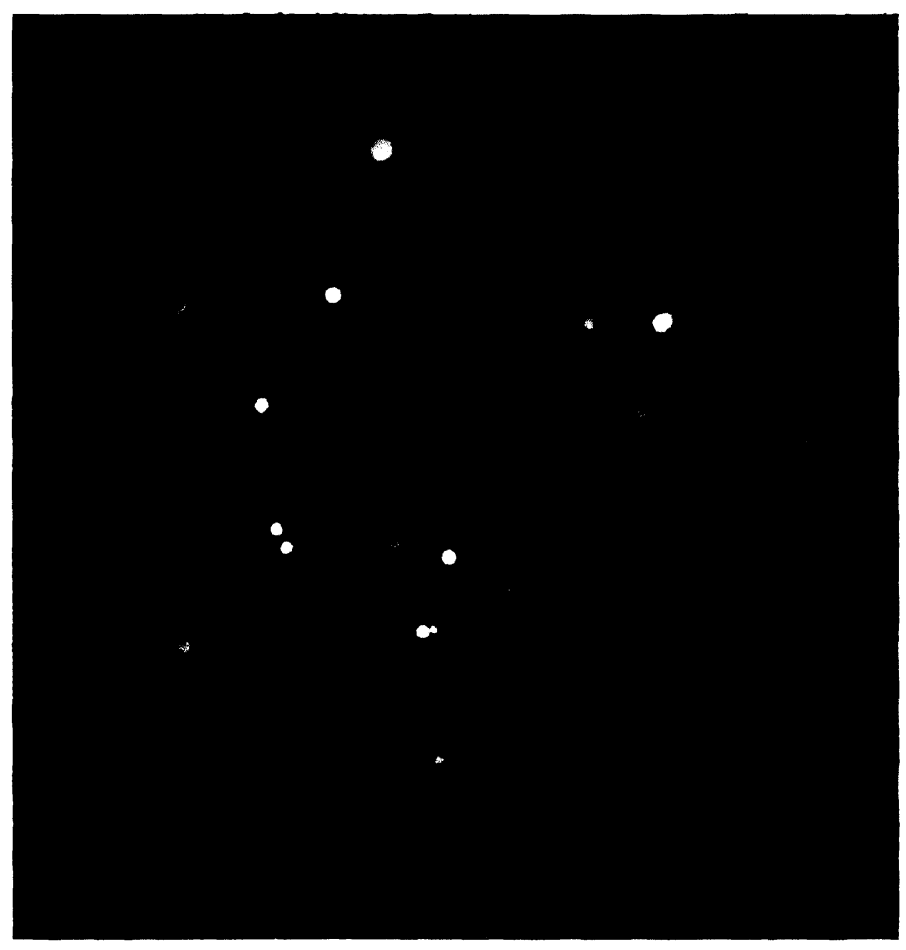

FIGURE 1. Combined ROSAT PSPC/HRI image of the Lockman Hole region. The HRI sources are shown in green colour. The red and blue colours indicate PSPC sources in the $0.1-0.5 \mathrm{keV}$ and $0.5-2.4 \mathrm{keV}$ energy bands. The HRI sources appear less extended due to the better spatial resolution of the HRI ( $\sim 5$ arc-sec) compared with that of the PSPC $(\sim 20$ arc-sec $)$. The field size is about 30 arc-min.

PSPC exposure, a $205 \mathrm{ksec}$ ROSAT HRI raster scan and a series of HRI images of a fixed area totalling $1112 \mathrm{ksec}$ (see Fig. 1). The ROSAT Deep Survey (RDS) based on the PSPC image includes a statistically complete sample of $50 \mathrm{X}$-ray sources with fluxes in the $0.5-2.0 \mathrm{keV}$ band larger than 5.5.10-15 $\mathrm{erg} \mathrm{cm}^{-2} \mathrm{~s}^{-1}$ (Hasinger et al. 1998). The optical identification of these faint X-ray sources has shown that over $75 \%$ of the RDS sources are AGNs (Schmidt et al. 1998). This study identified a much larger fraction of AGNs than did any previous X-ray survey (Boyle et al. 1995, Georgantopoulos et al. 1996, Bower et al. 1996 and McHardy et al. 1998).

The HRI images are the basis for the Ultradeep HRI Survey (Hasinger et al. 1999), which reaches now a surface density of $\sim 1000$ sources $\operatorname{deg}^{-2}$ at $10^{-15} \mathrm{erg}$ $\mathrm{cm}^{-2} \mathrm{~s}^{-1}$. At this flux limit 70-80\% of the soft X-ray background has been resolved into discrete sources. Both surveys contain in total $91 \mathrm{X}$-ray sources with fluxes in 
the $0.5-2.0 \mathrm{keV}$ band larger than $1.2 \cdot 10^{-15} \mathrm{erg} \mathrm{cm}^{-2} \mathrm{~s}^{-1}$.

Here we present the optical identification status of the ROSAT Deep Surveys in the Lockman Hole. We will discuss the population of red sources, which we have analyzed in the RDS. Finally, we compare the emission line properties of the RDS AGNs with those of several optical/UV and X-ray selected AGN samples.

\section{IDENTIFICATION OF THE ROSAT DEEP/ULTRADEEP SURVEYS}

The optical identification of the counterparts of most X-ray sources was obtained during the last five years using the Low Resolution Imaging Spectrometer (LRIS: Oke et al. 1995) on the Keck I and II telescopes on Mauna Kea, Hawaii. R-band images of the Lockman Hole field were taken to identify the faint optical counterparts $(R>24 \mathrm{mag})$ of the weakest X-ray sources. Fig. 2 shows the finding charts of some RDS sources. The HRI error circle is always smaller than the PSPC circle due to the higher spatial resolution of the ROSAT HRI detector as mentioned before. The HRI detection was crucial for the optical identification effort, because we found in nearly all cases a unique optical object inside the HRI error circle. Confusion in some RDS sources is resolved by the HRI, where the PSPC image shows only a single source (Schmidt et al. 1998, Lehmann et al. 2000a; hereafter Paper III).

The majority of optical spectra were taken with the LRIS instrument on the Keck I and II telescopes in the long-slit and multi-slit mode. The typical integration times were $1800 \mathrm{sec}$ for long-slit spectra and $3600 \mathrm{sec}$ for multi-slit masks. The wavelength coverage of the long-slit spectra is between 3800 and $8200 \AA$ using a 300 lines $\mathrm{mm}^{-1}$ grating. The wavelength range of the multi-slit spectra depends on the position of the objects on the masks (typical 4500-8900 $\AA$ ). The resolution of the spectra is about $\sim 10-15 \AA$ (FWHM). The high signal to noise Keck spectra are very important to identify the faint optical counterparts of the $\mathrm{X}$-ray sources.

For several relatively bright objects we have obtained spectra with the 4-Shooter spectrograph (Gunn et al. 1987) at the 5-m Hale telescope. Using a $1.5 \times 100^{\prime \prime}$ slit and a 200 lines $\mathrm{mm}^{-1}$ transmission grating the spectra cover a wavelength range of $4500-9500 \AA$ at a resolution of $25 \AA$. The optical spectra of some bright stars were taken with the Boller \& Chivens Cass Twin spectrograph at the 3.5-m telescope on Calar Alto. A 1.5" wide slit and the 600 lines $\mathrm{mm}^{-1}$ transmission grating (T13) was used resulting in a wavelength coverage of $3500-5500 \AA$ and a resolution of about $4.4 \AA$.

In Fig. 2 we present the Keck spectra of the counterparts of faint X-ray sources, which are contributors to the soft X-ray background (type I AGNs, type II AGNs and groups/clusters of galaxies). The flux scale is normalized so that one count corresponds to an $\mathrm{AB}$ magnitude $28\left(\mathrm{f}_{\nu}=2.29 \cdot 10^{-31} \mathrm{erg} \mathrm{s}^{-1} \mathrm{~cm}^{-2} \mathrm{~Hz}^{-1}\right)$. The location of typical AGN emission lines (eg. Ly $\alpha$, C IV, C III], Mg II) and galaxy absorption lines ( $\mathrm{Ca} \mathrm{H}+\mathrm{K} \lambda 3934 / 3968, \mathrm{CH} \mathrm{G} \lambda 4304, \mathrm{Mg}$ I $\lambda 5175, \mathrm{Na}$ I $\lambda 5890)$ are overplotted. For the whole set of optical spectra of RDS sources see Paper III.

The existence of broad emission lines reveals the AGN nature of many faint optical counterparts (eg. 37G in Fig.2). But the optical spectra of several objects 

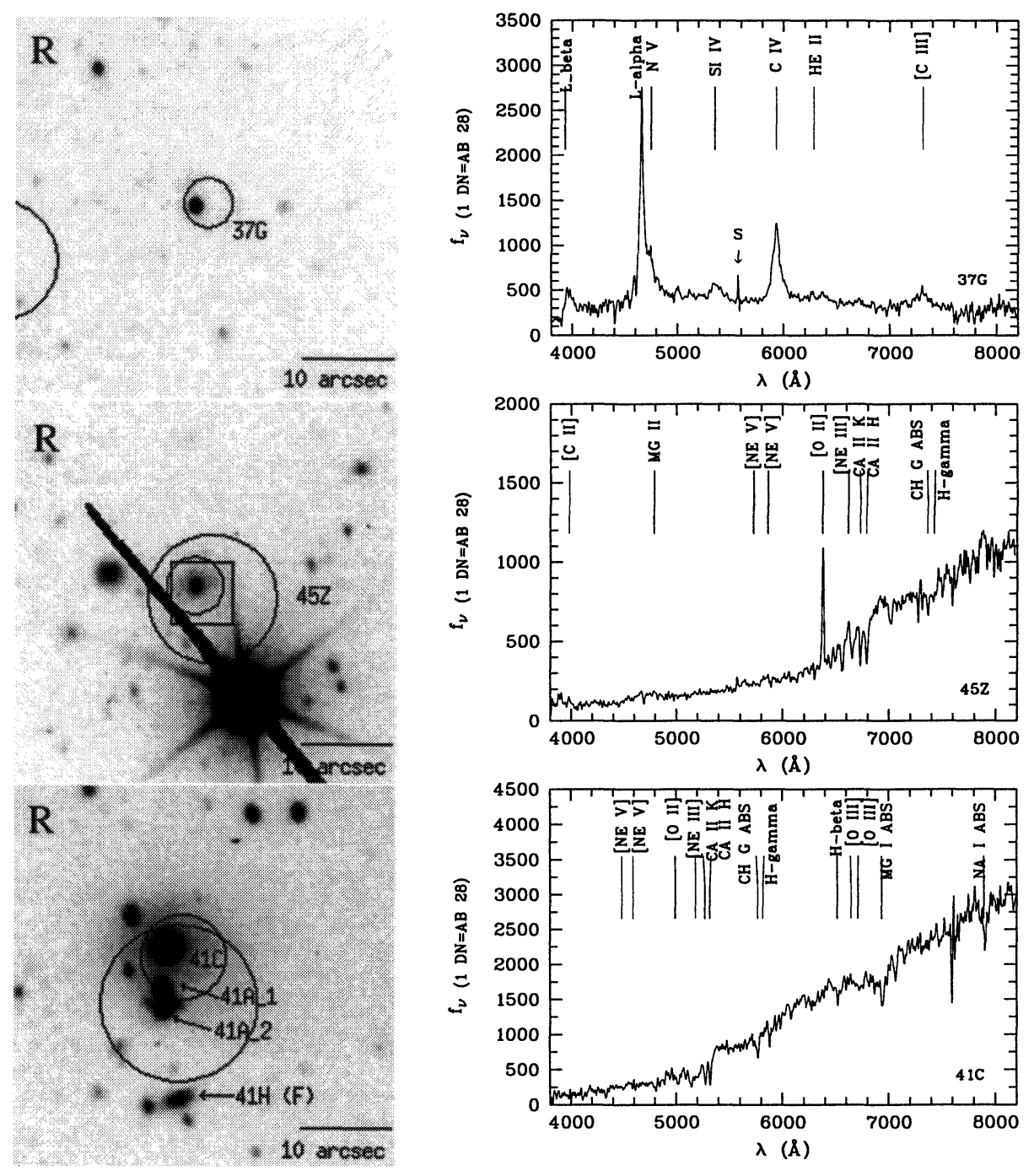

FIGURE 2. Examples of Keck $R$-band images and low-resolution spectra of optical counterparts (37G - type I AGN, 45Z - type II AGN, $41 \mathrm{C}$ - a member of a galaxy group). North is up, and east to the left. The small and large circles show the ROSAT HRI and PSPC error circles ( $80 \%$ error radius). The $2 \sigma$ error box indicates a VLA $20 \mathrm{~cm}$ detection (DeRuiter et al. 1997). Residuals of night sky emission lines are indicated with "s". 
contain no broad emission lines. Some of them show high ionization [Ne V] $\lambda 3426$ emission lines, typical for AGNs. Without these high quality Keck spectra and the accurate HRI positions they may have been identified with narrow-line X-ray galaxies (NLXG) as found in several other less deep soft X-ray surveys (see Boyle et al. 1995, Geogantopoulous et al. 1996, McHardy et al. 1998). Furthermore two of the RDS sources have X-ray luminosities in the $0.5-2.0 \mathrm{keV}$ energy band above $10^{43} \mathrm{erg} \mathrm{s}^{-1}$, show relatively strong [Ne III] $\lambda 3869$ emission lines, which are typically quite strong in Seyfert galaxies and quasars (Schmidt et al. 1998). The problem of missidentification of faint X-ray sources is discussed in more detail in Paper III.
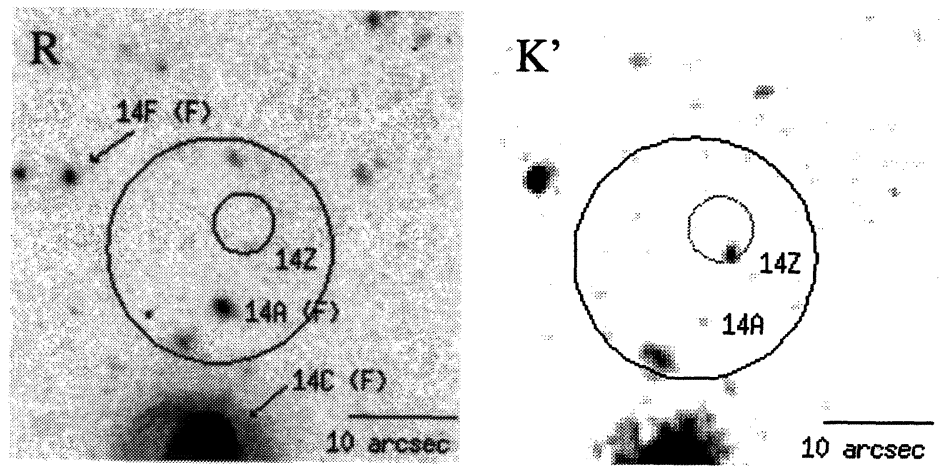

FIGURE 3. Keck $R$ and Calar Alto Omega-Prime $K^{\prime}$ images of source 14. The counterpart $14 \mathrm{Z}$ is clearly visible on the $K^{\prime}$ image (inside the HRI error circle), whereas it is only marginal detected on the $R$ image $(\sim 25.5 \mathrm{mag})$ resulting in a large $R-K^{\prime}$ colour of 5.4 mag. Field galaxies with known redshift are marked with $"(\mathrm{~F})$ ".

Some X-ray sources exhibit only a very faint $(R \sim 25 \mathrm{mag})$ or no optical counterpart at the limiting $R$-magnitude of 25.5 , which is clearly below the spectroscopic limit of our survey $(R \sim 23.5 \mathrm{mag})$. Therefore we started a deep broad-band $\mathrm{K}^{\prime}$ $(1.9244-2.292 \mu \mathrm{m})$ survey of the Lockman Hole region with the Omega-Prime camera (Bizenberger et al. 1998) on the Calar Alto 3.5-m telescope. About half of the Ultradeep HRI Survey area is already covered. The camera uses a $1024 \times 1024$ pixel $\mathrm{HgCdTe}$ HAWAII array with an image scale of $0.396 \mathrm{arcsec} /$ pixel, and covers a field-of-view of $6.7 \times 6.7 \mathrm{arcmin}$. Point sources are well detected at the $5 \sigma$ level at $\mathrm{K}^{\prime}=19.7 \mathrm{mag}$ in a $45 \mathrm{~min}$ (net) exposure.

$K^{\prime}$ and $R$ images are presented in Fig. 3 for the RDS source 14. A faint $K^{\prime}$ source is recognizeable within the HRI error circle $\left(K^{\prime}=19.6 \mathrm{mag}\right)$, whereas in the $R$-band the source is only marginally detected. The resulting $R-K^{\prime}$ colour is about $\sim 5.4$ mag. The RDS contains a further source with such a large $R-K^{\prime}$ colour (84Z: $R-K^{\prime} \geq 6.2$ ). The most probable identifications of such red objects are either with high redshift $(\mathrm{z} \geq 1)$ ellipticals or dust-reddened starbursts or AGNs. Since the source 14 has a relatively hard spectrum (Schmidt et al. 1998) we prefer the 
identification with a quasar reddened by absorption, since no additional red object is seen close to it in the $\mathrm{K}^{\prime}$ image (the object $14 \mathrm{~A}$, inside the PSPC error box, but outside the HRI circle, is a narrow emission line galaxy (NELG) at $z=0.546$, which is typical for the population of field galaxies (see Paper III).

There are several examples for faint X-ray sources identified with red objects (eg. Newsam et al. 1997). In our total sample of $91 \mathrm{X}$-ray sources we found that all X-ray counterparts with red colours $\left(R-K^{\prime}>4.5\right)$ are either members of high redshift clusters of galaxies $(\mathrm{z}>1)$ or obscured AGNs at various redshifts. Recently, we have detected a number of red galaxies (Lehmann et al. 2000b) indicating one of the most distant $\mathrm{X}$-ray selected clusters of galaxies with a gravitationally lensed arc (Hasinger et al. 1999b).

\begin{tabular}{|l|c|c|}
\hline objects class & number of object & total content in [\%] \\
\hline AGNs type I & 54 & 59.3 \\
AGNs type II & 15 & 16.5 \\
galaxies & 1 & 1.1 \\
groups/clusters & 10 & 11.0 \\
stars & 5 & 5.5 \\
unidentified sources & 6 & 6.6 \\
\hline total & 91 & \\
\hline
\end{tabular}

TABLE 1. Spectroscopic identification status of the ROSAT Deep Surveys.

In Table 1. we present the identification status of the $91 \mathrm{X}$-ray sources above the limiting flux of $1.2 \cdot 10^{-15} \mathrm{erg} \mathrm{cm}^{-2} \mathrm{~s}^{-1}$ in the $0.5-2.0 \mathrm{keV}$ energy band. The large majority of the $\mathrm{X}$-ray sources in this sample $(\sim 75 \%)$ turned out to be AGNs. Most of these objects are classified as AGNs type I (quasars and Seyfert galaxies), because they exhibit at least one broad emission line in their optical spectra. Among these objects is the most distant $\mathrm{X}$-ray selected quasar $(\mathrm{z}=4.45)$ found to date (Schneider et al. 1998). A non-negligible fraction of AGNs is classified with AGNs type II showing only narrow emission lines or broad $\mathrm{H} \alpha / \mathrm{H} \beta$ lines with a large Balmer decrement. For some of them only the existence of high excitation [Ne V] emission lines and/or the high X-ray luminosity $\left(\mathrm{L}_{X}>10^{43} \mathrm{erg} \mathrm{s}^{-1}\right)$ in the $0.5-2.0 \mathrm{keV}$ energy band reveal the AGN nature of these objects. From the nearly complete (95\%) identification of our ROSAT Deep Surveys we find no indication for new classes of NLXG or of galactic CVs (Maoz \& Grindlay 1995), which have been claimed to dominate the soft X-ray counts at fainter fluxes (cf. Paper III).

\section{POPULATION OF RED AGNS IN THE RDS}

The optical spectra of most RDS AGNs with redshift below 1.1 show absorption lines typical for galaxies, eg. Ca $\mathrm{H}+\mathrm{K} \lambda 3934 / 3968, \mathrm{CH} \mathrm{G} \lambda 4304, \mathrm{Mg}$ I $\lambda 5175, \mathrm{Na}$ I $\lambda 5890$. This suggests a significant contribution by the host galaxy in the optical wavelength range. Therefore we have measured the break index $\mathrm{D}(4000)$ in order 

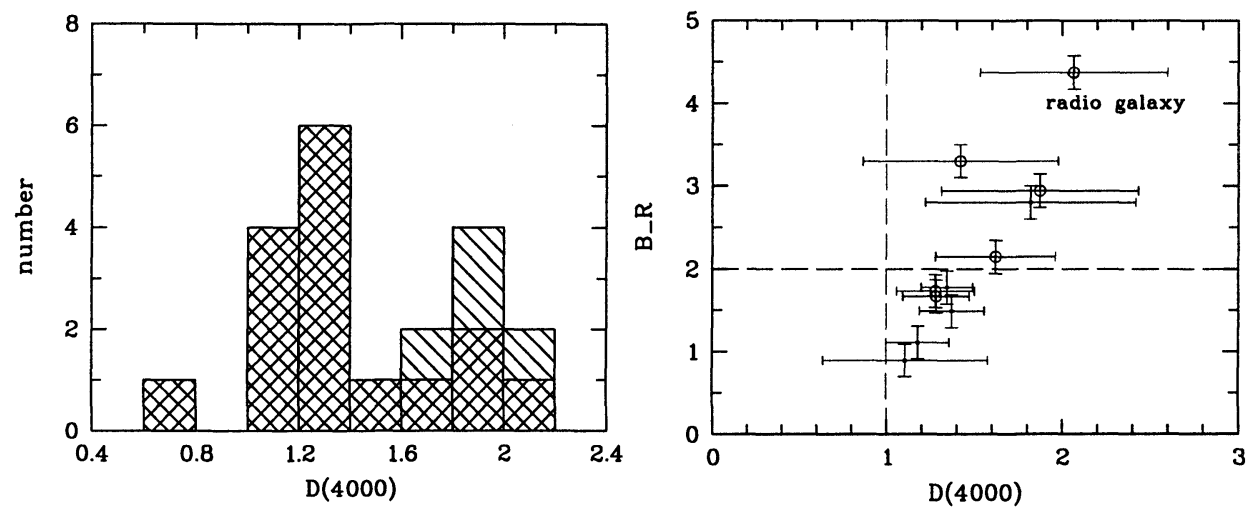

FIGURE 4. a) Distribution of the D(4000) index of 16 RDS AGNs. In addition, the values of the three group members $41 \mathrm{C}, 58 \mathrm{~B}, 67 \mathrm{~B}(\mathrm{~s})$ and of the RDS galaxy $53 \mathrm{~A}$ are overplotted with a different hatch. b) $B-R$ colour derived from the optical spectra vs. the $\mathrm{D}(4000)$ index for 11 RDS AGNs. The dots with error bars are the broad-line AGNs, whereas the circles indicate the narrow-line AGNs. In the other cases the optical spectra could not be used to obtain the $B-R$ values. The horizontal line marks the region of red AGNs $(B-R>2.0)$. The AGNs right of the vertical line with $\mathrm{D}(4000)>1$ show a continuum component originated from the host galaxy.

to have an estimate of the main stellar population in the host galaxies. Early-type galaxies show a pronounced break around $4000 \AA$ in their optical spectra. The $4000 \AA$ break index $\mathrm{D}(4000)$ is defined by Bruzual (1983). Due to the wavelength range of our spectra we were able to derive the $\mathrm{D}(4000)$ values for all objects with redshift between 0.01 and 0.93 .

About $63 \%$ of the RDS AGNs for which the $\mathrm{D}(4000)$ index could be derived from the optical spectra, have $\mathrm{D}(4000)>1$ at more than 1 sigma level (cf. Paper III). One object, whose spectrum shows a very blue continuum, is the only AGN with $\mathrm{D}(4000)$ $<1$, consistent with a power law continuum $\left(\mathrm{f}_{\nu}=\nu^{\alpha}\right.$ with $\left.\alpha<0\right)$. The histogram of the $\mathrm{D}(4000)$ index of the RDS AGNs is shown in Fig. 4a. This distribution peaks at $\mathrm{D}(4000) \sim 1.2$, with most of the objects in the range $1.0 \leq \mathrm{D}(4000) \leq 1.4$, plus some objects at larger values $(1.6 \leq \mathrm{D}(4000) \leq 2.0)$. These two ranges of $\mathrm{D}(4000)$ correspond to galaxies with a sustained amount of star formation and galaxies with a dominant old stellar population, respectively. The $\mathrm{D}(4000)$ values of the three RDS group members $(41 \mathrm{C}, 58 \mathrm{~B}$ and $67 \mathrm{~B})$ are all consistent with those of elliptical galaxies.

As a comparison, the mean $\mathrm{D}(4000)$ values measured from the Canada-France Redshift Survey (CFRS; Lilly et al. 1995) in a redshift interval similar to that of our objects $(\mathrm{z}<1)$ are 1.43 for 256 emission line galaxies and 1.92 for 85 "quiescent" galaxies, defined as galaxies with no detected [OII] line (from Table 1 in Hammer et al. 1997). Similar average values (1.40 and 1.83 for emission line galaxies and no-emission line galaxies) have recently been measured from the large, more local 
$(\mathrm{z}<0.15)$ Stromlo-APM Survey (Tresse et al. 1999).

The $\mathrm{D}(4000)$ values do not appear to correlate either with the absolute magnitudes of the AGNs, which cover the range $-23.9<\mathrm{M}_{V}<-20.7$, or with the presence of broad emission lines. In order to check if the $\mathrm{D}(4000)$ index is correlated with the colours of the objects we have determined the $B-R$ colours of the RDS AGNs. Unfortunately we have no good photometric catalogue yet in the $B$-band. Therefore we have derived the $B-R$ colour from the optical spectra for those objects, where the spectra were taken at the paralactic angle to avoid atmospheric refraction. Fig. 4.b shows the $B-R$ colours of 11 RDS AGNs versus the D(4000) index. Despite of the relatively large errors and the small number of objects there is a trend that the $B-R$ colour of the AGNs increases with increasing $\mathrm{D}(4000)$ value. Five of the 11 RDS AGNs have red colours $(B-R \geq 2.0)$. The AGNs with the largest $B-R$ value is a radio galaxy, were the optical spectrum reveals an elliptical galaxy. All of these red AGNs have relatively large $\mathrm{D}(4000)$ values indicating a significant contribution of the host galaxy to the continuum emission. The red colour of some RDS AGNs could therefore originate from their host galaxies.

Recently, Fiore et al. (1999) have found two red AGNs from the first optical identification of the BeppoSAX HELLAS Survey in the 5-10 keV energy band. Their optical spectra are as well dominated by starlight.

\section{EMISSION LINE PROPOERTIES OF RDS AGNS}

In order to derive a reliable optical identification of the RDS X-ray sources we have determined the emission line properties of the most prominent AGN and galaxy emission lines. Due to the large redshift range of the RDS AGNs from 0.080 to 2.832 with its mean redshift of $\langle z\rangle=1.2$ (cf. Fig 5. in Paper III), any given emission line is present only in a small number of objects.

The emission lines have been measured by fitting with single or double Gaussian profiles applying the Levenberg-Marquardt algorithm (Press et al. 1992). The Levenberg-Marquardt algorithm provides one sigma errors of the parameters. In some cases a single Gaussian gave a poor fit to the data, so an additional Gaussian was used. From the best set of parameters we have calculated the redshift, the rest frame $\mathrm{EW}_{\text {rest }}$ in $\AA$ and the FWHM in $\mathrm{km} \mathrm{s}^{-1}$. The redshift of each object was derived as the mean value from the strongest features (eg. Mg II $\lambda 2798$, [O II] $\lambda$ $3727)$. Its mean error is around $\sim 0.001$. The FWHM has been corrected for intrumental resolution. The FWHM and $\mathrm{EW}_{\text {rest }}$ of all emission lines of the RDS AGNs are given in Paper III.

The emission lines are divided into narrow emission lines $\left(<1500 \mathrm{~km} \mathrm{~s}^{-1}\right)$ and broad emission lines $\left(>1500 \mathrm{~km} \mathrm{~s}^{-1}\right)$. In addition, a few spectra required very broad line components $\left(>8000 \mathrm{~km} \mathrm{~s}^{-1}\right)$ of the semi-permitted line C III] $\lambda 1908$ and of the permitted lines C IV $\lambda 1548$ and Mg II $\lambda 2798$.

We compared the mean FWHM of the RDS AGNs with the properties of the UV/optically selected, low-redshift quasar sample $(\langle z\rangle=0.54)$ from Green 1996 and with the FWHM of a QSO sample at intermediate redshift $(0.9 \leq z \leq 2.2)$ from 
Brotherton et al. 1994. In addition we used for comparison the emission line properties derived from two X -ray surveys: the RIXOS AGN X-ray sample with $\langle z\rangle=0.82$ (Puchnariwicz et al. 1997) and the AGN data of the Cambridge-Cambridge ROSAT Serendipity Survey (CRSS) of Boyle et al. (1997), which has a mean redshift of $\langle z\rangle=0.88$. In general the mean FWHM of the RDS AGN broad emission lines (Si IV $\lambda 1397, \mathrm{C}$ IV $\lambda 1548, \mathrm{C}$ III] $\lambda 1908, \mathrm{Mg}$ II $\lambda 2798, \mathrm{H} \beta \lambda 4861$ and $\mathrm{H} \alpha \lambda 6563$ ) are consistent with those values found for other X-ray selected AGN samples as well as with the data obtained from the mentioned optical/UV selected AGN samples. Only the FWHM of the broad Ly $\alpha \lambda 1216$ emission lines (detected only in two AGNs) are smaller than the mean FWHM of the Green quasar sample.

For a comparison of the mean $\mathrm{EW}_{\text {rest }}$ of RDS AGNs we have used in addition the range of the mean EW derived for several emission line surveys (Osmer 1980, Vaucher \& Weedman 1980) and optical/radio quasar surveys (Neugebauer 1979, Richstone \& Schmidt 1980, Oke \& Korycanski 1982, Wampler et al. 1984, Tytler et al. 1987) given in Schmidt et al. (1986) and the sample of high-redshift, bright QSO $\langle z\rangle=1.6$ from Steidel \& Sargent (1991). Further, we have calculated the mean EW (neglecting uncertain values) from the X-ray selected AGN sample $(\langle z\rangle=0.19)$ of Stephens 1989.

The mean RDS AGN EW of the broad emission lines are consistent with the data from several X-ray selected AGN samples at lower mean redshift (eg. RIXOS: $\langle z\rangle=0.8$ and CCRS: $\langle z\rangle=0.9)$ compared to its mean redshift of $\langle z\rangle=1.2$. Further they are in general in good agreement with the mean EW from UV/optical selected AGN samples at low and high redshift. Although our results are derived from a small number of emission lines they seem to indicate in comparison with the other samples nearly the same properties of AGN broad emission lines.

\section{ACKNOWLEDGEMENTS}

We acknowledge the support in part by NASA grants NAG5-1531 (M.S.), NAG8794, NAG5-1649, and NAGW-2508 (R.G.), and NSF grant AST-9509919 (D.S.). G.H. acknowledges the grant FKZ 50 OR 99080 by the Deutsches Zebtrum für Luftund Raumfahrt e.V. (DLR). G.Z. acknowledges partial support by the Italian Space Agency (ASI) under contract ARS-96-70 and the Italian Ministry for University and Research (MURST) under grant Cofin 98-02-32.

\section{REFERENCES}

Bizenberger P., McCaughrean M. J., Birk C., et al., 1998, Omega Prime: the wide-field nearinfrared camera for the $3.5 \mathrm{~m}$ telescope of the Calar Alto Observatory. In A. M. Fowler, ed., Infrared astronomical instrumentation, SPIE vol. 3354, p. 825

Bower R.G., Hasinger G., Castander F.J., et al., 1996, MNRAS 281, 59

Boyle B.J., McMahon R.G., Wilkes B.J., Elvis M., 1995a, MNRAS 272, 462

Boyle B.J., Wilkes B.J., Elvis M., 1997, MNRAS 285, 511

Brotherton M.S., Wills B.J., Steidel C.C., Sargent W.L.W., 1994, APJ 423, 131 
Bruzual A.G., 1983, ApJ 273, 105

De Ruiter H.R., Zamorani G., Parma P., et al., 1997, A\&A 319, 7

Georgantopoulos I., Stewart G.C., Shanks T., et al., 1996, MNRAS 280, 276

Giacconi R., Gursky H., Paolini F.R., et al., 1962, Phys. Rev. Lett. 9, 439

Green P.J., 1996, ApJ 467, 61

Gunn J.E., Carr M.L., Danielson G.E., et al., 1987, Opt. Eng. 26, 779

Fiore F., La Franca F., Giommi P., Elvis M., Matt G., et al., 1999, MNRAS 306, 55

Hammer F., Flores H., Lilly S.J., et al., 1997, ApJ 481, 49

Hasinger G., Burg R., Giacconi R., et al., 1993, A\&A 275, 1 (erratum A\&A, 291, 348)

Hasinger G., Burg R., Giacconi R., et al., 1998, A\&A 329, 482 (Paper I)

Hasinger G., Lehmann I., Giacconi R., et al., 1999a, In Proceedings of the Symposium "Highlights

in X-ray Astronomy in honour of Joachim Trümper's 65th birthday", eds. B.Aschenbach \& M.J.Freyberg, MPE Report 272, p. 199

Hasinger G., Giacconi R., Gunn J. E., et al., 1999b, A\&A 340L, 27 (Paper IV)

Lockman F.J., Jahoda K., McCammon D., 1986, ApJ 302,432

Lehmann I., Hasinger G., Schmidt M., et al., 2000a, A\&A 354, 35 (Paper III)

Lehmann I., Hasinger G., Giacconi R., et al., 2000b, In Proceedings of the VLT Opening Symposium, held at Antofagasta, Chile, 1-4 March 1999, eds. J. Bergeron \& A. Renzini, Springer, p. 121

Lilly S.J., Le Fèvre O., Crampton D., Hammer F., Tresse L., 1995, ApJ 455, 50

Maoz E., Grindlay J.E., 1995, ApJ 444, 183

McHardy I., Jones L.R., Merrifield M.R., et al., 1998, MNRAS 295, 641

Neugebauer G., Oke J.B., Becklin E.E., Matthews K., 1979, ApJ 230, 79

Newsam A.M., McHardy I.M., Jones L.R., Maison K.O., 1997, MNRAS 292, 378

Oke J.B., Cohen J.G., Carr M., et al., 1995, PASP 107, 375

Osmer P.S., 1980, ApJ Suppl. 42, 523

Puchnarewicz E.M., Mason K.O., Carrera F.J., et al., 1997, MNRAS 291, 177

Press H.W., Teukolski S.A., Vetterling W.T., Flannery B.P., 1992, Receipes (in FORTRAN), sec. edition, Cambridge Univ. Press,

Richstone D.O. \& Schmidt M., 1980, ApJ 235, 377

Schmidt M., Schneider D.P., Gunn J.E., 1986, ApJ 306, 411

Schmidt M., Hasinger G., Gunn J.E., et al., 1998, A\&A 329, 495 (Paper II)

Schneider D.P., Schmidt M., Hasinger G., et al., 1998, AJ, 115, 1230

Steidel C.C., Sargent W.L.W., 1991, ApJ 382, 433

Stephens S.A., 1989, AJ 97, 10

Tresse L., Maddox S., Loveday J., Singleton C., 1999, MNRAS 310, 262

Vaucher B.G. \& Weedman D.W., 1980 ApJ 240, 10

Wampler E.J., Gaskell C.M., Burke W.L., Baldwin J.A., 1984, ApJ 276, 403 